\title{
Lumen
}

Selected Proceedings from the Canadian Society for Eighteenth-Century Studies

\section{Robert Challe, témoin suspect de la prise de Chédabouctou}

\section{Driss Aissaoui}

Volume 27, 2008

North America at the Crossroads of European Cultures in the Eighteenth Century

L’Amérique du Nord au Carrefour des cultures au XVIII ${ }^{\mathrm{e}}$ siècle

URI : https://id.erudit.org/iderudit/1012046ar

DOI : https://doi.org/10.7202/1012046ar

Aller au sommaire du numéro

Éditeur(s)

Canadian Society for Eighteenth-Century Studies / Société canadienne d'étude du dix-huitième siècle

ISSN

1209-3696 (imprimé)

1927-8284 (numérique)

Découvrir la revue

Citer cet article

Aissaoui, D. (2008). Robert Challe, témoin suspect de la prise de Chédabouctou. Lumen, 27, 1-11. https://doi.org/10.7202/1012046ar

Copyright (C Canadian Society for Eighteenth-Century Studies / Sociéte canadienne d'étude du dix-huitième siècle, 2009
Ce document est protégé par la loi sur le droit d'auteur. L'utilisation des services d'Érudit (y compris la reproduction) est assujettie à sa politique d'utilisation que vous pouvez consulter en ligne.

https://apropos.erudit.org/fr/usagers/politique-dutilisation/ 


\section{Robert Challe, témoin suspect de la prise de Chédabouctou}

«Un individu quel qu'il soit n'est pas un témoin digne de foi en sa propre cause», affirme Georges Gusdorf dans Les Écritures $d u$ moi ${ }^{1}$. C'est dire que la complaisance à soi constitue un préjugé qui pèse souvent sur la littérature personnelle. Des Mémoires du Cardinal de Retz à ceux de Goethe en passant par les Confessions de Rousseau, les écritures du moi, dans la grande variété qui les caractérise, n'échappent que rarement aux accusations de mensonge et de gauchissement de la réalité objective. Nombreux, en effet, sont les historiens d'eux-mêmes qui s'appliquent à mêler le vrai au faux. Jugeant que le discours sur soi n'est pas une reproduction littérale des jours anciens, ces écrivains en aveu n'hésitent pas à transformer les données de la réalité objective au gré de mobiles personnels.

Loin de tenir la promesse qu'il formule aux abords de son Journal de donner un démenti au dicton «il fait bon mentir à qui vient de loin», Robert Challe ne fait que grossir les rangs de ces écrivains en aveu qui se plaisent à infléchir le cours de l'histoire, à chaque fois que le détournement des faits leur procure un quelconque prestige. Sous la plume de Challe, le témoignage historique se fait souvent par des détours dans le domaine de la fiction. Si certaines affirmations suspectes échappent à toute vérification, toutefois, il en est autrement pour celles auxquelles les documents historiques apportent un complément d'information et permettent de surprendre Challe en flagrant délit $\mathrm{d}^{\prime}$ « embellissement » de son odyssée personnelle. Le récit de l'aventure acadienne illustre bien la propension qu'a cet historien de lui-même à mêler ce qui a été et ce qui aurait pu être.

1 Georges Gusdorf, Lignes de vie 1. Les écritures du moi, Paris, Odile Jacob, 1991, p. 23. 
Cet épisode acadien, qui s'étend de 1682 à 1688, Challe y fait écho à la fois dans les Mémoires et dans le Journal. Bien qu'elles soient souvent complémentaires, les indications données sur cette aventure dans les deux textes personnels se révèlent parfois contradictoires, permettant de douter de l'authenticité du témoignage de Challe concernant cette période de sa vie. Sur un épisode au moins de la déconfiture acadienne, à savoir la prise de Chédabouctou, le texte des Mémoires prend ses distances par rapport à celui du Journal. De cet événement qu'il lie directement à sa propre ruine, Challe donne deux témoignages distincts. Si les informations fournies conjointement par les deux textes révèlent la profonde connaissance qu'a l'écrivain de la situation des colonies françaises d'outre-mer, les incohérences que fait apparaître la comparaison entre la version du Journal et celle des Mémoires rendent encore une fois les propos de Challe suspects. Rappelons les faits tels qu'ils sont présentés, dans le Journal d'abord :

Notre fort était bien garni de trente canons bien montés, avec les munitions nécessaires, tant de guerre que de bouche. Il fut pourtant, grâce à la vigilante conduite du gouverneur et des officiers, pris un beau matin, 23 juin 1687, par un seul détachement d'Anglais venu à travers les bois depuis la Hève, où ils m'avaient pris, moi, avec une barque chargée de pour plus de cinquante mille écus de castors, \& parce que je m'étais fait tuer dix-neuf hommes, et que je ne m'étais rendu que blessé à quatre endroits, ils me conduisirent à Baston, où ils voulaient, disaient-ils, me faire pendre [...] Il [le gouverneur] fut pris dans son lit [...] les autres [...] furent tout pris comme lui, les portes du fort étant ouvertes. Les trois vaisseaux qui travaillaient à la pêche furent pris sans résistance. Le fort fut détruit rez-pied, rez-terre : le canon fut mis sur les vaisseaux; et un mois environ après, je les vis arriver à Baston, où j'étais, avec pour plus de cent mille francs de poisson, tant vert que sec, \& pour plus de cinq cent mille francs de pelleteries ${ }^{2}$.

À cette version du Journal, les Mémoires proposent la réplique suivante :

Les Anglais envoyèrent au fort de Chédabouctou des gens affidés qui remarquèrent le peu de fortifications qu'il y avait, que les canons n'étaient pas même montés. Ils remarquèrent les chemins par terre, et de plus ils apprirent qu'on

2 Robert Challe, Journal d'un voyage fait aux Indes Orientales, édition critique établie par Frédéric Deloffre et Melahat Menemencioglu, Paris, Mercure de France, 1979, p. 215. 
n'y faisait ni guet ni garde, parce que La Boulaye gouverneur envoyait tout le monde à la pêche, ils vinrent à Canseau dans un temps où on ne les attendait nullement, prirent les vaisseaux qu'ils trouvaient dont trois appartenaient à la Compagnie, arrêtèrent tout le monde, et entrant dans le golfe de Chédabouctou, ils mirent deux cents hommes à terre à trois lieues du fort, et à travers les bois sans rencontrer qui que ce fût, ils vinrent se coucher à terre à la porte du fort, et à la pointe du jour lorsqu'on ouvrit cette porte, ils entrèrent l'épée et le pistolet à la main. Il n'y eut qui que ce soit de blessé parce que personne ne fit de résistance. Ils entrèrent dans la chambre du gouverneur qu'ils trouvèrent endormi dans son lit, sans doute de la fatigue qu'il avait prise avec une sauvage qui fut trouvée couchée avec lui. [...] Le magasin que j'avais laissé bien garni, servit de proie aux Anglais aussi bien que les pelleteries qui avaient été traitées pendant l'hiver, et le poisson qu'on avait pêché pendant le printemps et partie de l'été. Tout fut conduit à Baston par nos propres vaisseaux, sans oublier les canons du fort que les Anglais rasèrent rez pied rez terre. ${ }^{3}$

La comparaison des deux comptes rendus donnés par Challe de la prise du comptoir de Chédabouctou fait surgir de multiples contradictions. Dans le Journal, Challe dit que les canons sont bien montés, alors que dans les Mémoires il affirme le contraire ${ }^{4}$. Sur ce point, l'écart entre les deux versions peut s'expliquer par les vingt-cinq années qui séparent la première rédaction du Journal et la composition des Mémoires. Pendant ce laps de temps, Challe a peut-être eu un supplément d'information qui l'aurait amené à modifier son témoignage. Soulignons à cet égard qu'il n'était pas à Chédabouctou au moment de l'assaut. Mais la discordance entre la version du Journal et celle des Mémoires n'est pas la seule à jeter un doute sur le témoignage de Challe. Les renseignements fournis par certains documents historiques font apparaître d'autres impertinences.

Dans la version du Journal, Challe nous apprend que la prise de Chédabouctou et son emprisonnement par les Anglais à La Hève ont eu lieu le 23 juin 1687. Or nous savons, grâce aux recherches de Jean

3 Robert Challe, Les Mémoires, publiés d'après les originaux avec de nombreux documents inédits par Frédéric Deloffre avec la collaboration de Jacques Popin, Genève, Droz, 1996, p. 387-389.

4 Notons au passage - mais ce n'est là qu'un fait anecdotique - que le nombre de femmes trouvées aux côtés de La Boulaye change : de deux dans le Journal, on passe à une seule dans les Mémoires. 
Mesnard, qu'il ne s'est pas rendu au Canada cette année-là ${ }^{5}$. Challe a dû se tromper de date. Peut-être a-t-il ajouté cet épisode pendant la refonte du Journal autour de 1721. L'attaque contre le comptoir de la compagnie de la pêche sédentaire a eu lieu un an plus tard, c'est-à-dire en l'été 1688. Un texte atteste la présence de Challe en Acadie en juillet $1688^{6}$. La deuxième correction qu' on pourrait apporter aux témoignages de Challe concerne la destruction du fort. Challe se trompe encore de date quand il prétend que ce comptoir fut rasé rez pied rez terre. Lors de l'inspection menée par Vincent Saccardy, ingénieur général du roi, le 4 septembre 1689, l'établissement existait encore ${ }^{7}$. Le fort n'a été détruit «rez-pied, rez-terre» que lors de la deuxième prise conduite par Cyprian Southack en 1690. Agissant sous les ordres de William Phips, ce corsaire anglais " s'empara du fort après s'être fait repousser par deux fois. Il mit le feu et fit sauter la réserve de poudre, anéantissant tout ${ }^{8}$ ».

Malgré les contradictions qu'ils comportent et les écarts qu'ils présentent par rapport à certains documents officiels, les récits de Challe constituaient la seule source d'information concernant cet épisode de l'histoire coloniale française. Mais un recueil de documents anciens découvert récemment présente un autre témoignage, direct celui-là, offrant un démenti aux affirmations de Challe. Il s'agit du récit donné par La Boulaye lui-même. Dans une lettre adressée à $M$. Denonville, gouverneur de l'Acadie, le commandant de Chédabouctou donne sa propre version des faits. Même si elle est relativement longue, elle mérite d'être reproduite, dans la mesure où elle fournit un éclairage nouveau sur cette affaire :

Le 10 août 1688, il serait venu à nous une chaloupe dans laquelle étaient deux hommes et l'équipage du capitaine Candé avec son chirurgien et trois autres hommes inconnus, lesquels se seraient jetés dans notre charon, le sabre et le pistolet à la main, et, cachant le pistolet, auraient crié: Tue, tue, bon quartier. Nous

5 Jean Mesnard, " Entre Mémoires et fiction : le thème acadien chez Robert Challe ", Travaux de littérature, vol. III, 1990, 297-323.

6 Ce document (The cartographic journal of Nova Scotia MS $2 \mathrm{n}^{\circ} 370$ ) se trouve aux Collections spéciales de la bibliothèque Killam de l'Université Dalhousie, à Halifax. Il est signé par un géographe français du nom de Lalanne. Décrivant sa mission qui consiste à explorer les routes maritimes et d'éventuels ports autour de la péninsule acadienne, ce dernier mentionne le nom de Challe.

7 Voir sur ce point la récente édition des Mémoires, op. cit., p. 389, à la note 292,34.

8 Marie-Laure Girou-Swiderski, « Le pilleur de Chédabouctou », article inédit, p. 11. L'étude m'a été communiquée par l'auteure elle-même, que je remercie. 
leur demandâmes ce que c'était. Ils dirent Messieurs, nous sommes Anglais; et par derrière, il arriva une autre chaloupe armée de six hommes et dirent: «Voilà notre capitaine.»

-Et bien Messieurs, dirent-nous, y a-t-il guerre?

Ils répondirent: «Nous sommes flibustiers. Nous avons besoin de vivres. Nous en voulons avoir, si ce n'est d'amitié, ce sera par force. Nous allions aller à l'habitation, mais nous en avons su que vous veniez, ce qui nous a fait attendre.» Et, en même temps, nous menant dans le navire du capitaine Candé qui était pris où le capitaine du forban nous laissa avec cinquante hommes de son équipage, lesquels nous firent beaucoup souffrir toute la nuit par leurs menaces et jurements, et particulièrement au Révérend Père Raphaël, contre lequel ils proférèrent une quantité d'imprécations en haine de la Foi Catholique Romaine.

[...] Le lendemain, le forban équipa cinq chaloupes armées de 38 hommes qui nous menèrent à l'habitation de Chédabouctou, où nous arrivâmes à minuit avec des jurements que, s'ils trouvaient la moindre résistance, ils mettraient tout à feu et à sang, ce que n'avait garde d'arriver, attendu que le peu de monde qui était à l'habitation était fatigué d'avoir travaillé pendant plus de trois semaines à décharger les marchandises et vivres venues tant de France que de Québec, lesquels ne songeant qu'à se reposer, n'appréhendaient aucune chose. ${ }^{9}$

Entre le récit de La Boulaye et le témoignage de Challe une similarité persiste : l'un et l'autre disent que le fort a été pris sans résistance. Mais les deux hommes ne s'accordent pas sur les raisons qui ont conduit à cette reddition. Si Challe l'explique par la poltronnerie du commandant, par son ignorance des stratégies défensives et par son attachement excessif aux plaisirs, La Boulaye invoque, quant à lui, l'effet de surprise et la fatigue, bien légitime, de ses hommes qui se sont affairés pendant plusieurs jours à vider la cargaison du bateau. À considérer les

9 "Pillage fait par les Anglois de La Nouvelle Angleterre ", dans Jean Blanchet, Collection de manuscrits contenant lettres, mémoires, et autres documents historiques relatifs à la Nouvelle-France: recueillis aux Archives de la province de Québec ou copiés à l'étranger, mis en ordre et édités sous les auspices de la Législature de Québec, etc., vol. 1, Québec, 1883, 428-429. En étudiant ce document, Raynald Laprise (voir note 11) ne savait pas qu'il apportait un éclairage nouveau sur cet épisode de l'histoire acadienne. Non seulement, il ne mentionne pas que les spécialistes de Challe ignoraient l'existence d'un tel témoignage, mais il utilise une édition des Mémoires à la fois contestée et dépassée, établie par Augustin Thierry en 1931. Or il existe une édition plus récente et plus représentative, établie par Frédéric Deloffre (op. cit.). 
deux témoignages pour eux-mêmes, il serait difficile de pencher vers l'un ou l'autre. Cette discorde est d'autant plus délicate à arbitrer que les deux témoins sont intéressés dans l'affaire qu'ils rapportent. Mais dès que des sources externes entrent en ligne de compte, le témoignage de La Boulaye paraît plus crédible que celui de Challe. En tout cas plusieurs documents officiels reprennent sa thèse.

En effet, si la version présentée par Denonville ${ }^{10}$ au secrétaire d'État Seigneley, qui est calquée sur celle de La Boulaye, peut avoir dissimulé certains faits incriminant le commandant de Chédabouctou, l'officier anglais Nicholson n'y a aucun intérêt. Par contre, Challe possédait une part des marchandises qui se trouvaient à Chédabouctou et dont s'emparèrent les flibustiers. Il est donc possible qu'il ait voulu régler ses comptes avec La Boulaye, qu'il rendait responsable, par son inertie et son manque de prévoyance, de la perte financière qu'il subit lors $\mathrm{du}$ raid de Peterson. Ce n'est donc pas en se fiant à une seule version, mais en colligeant plusieurs témoignages et en les confrontant avec différents documents d'archives qu'on peut établir la vérité, ou presque, sur ce qui s'est passé à Chédabouctou en ce mois d'août 1688.

\section{Reconstitution des faits}

Le recoupement de différents documents officiels a permis à un chercheur acadien de récapituler les faits relatifs à cet événement tels qu'ils se seraient réellement passés entre le 10 et le 15 août 1688. Notons que dans le compte rendu qu'il nous propose de cet événement, Raynald Laprise reprend, en modernisant leur style, les documents recueillis par Jean Blanchet dans sa Collection de manuscrits [...] relatifs à la Nouvelle-France.

Le 14 juillet, le navire nommé Le St-Louis, de 250 tonneaux, appartenant au sieur Claisson de La Rochelle, et fretté par la compagnie de l'Acadie, arriva à Chédabouctou où il déchargea tous les vivres et toutes les marchandises qu'il avait apportées, non seulement pour ce lieu où la pêche sédentaire est établie, mais aussi pour le Port Royal et le reste de l'Acadie. Le 7 août, ce navire et une barque de la Compagnie, chargés de vivres et de marchandises destinées

10 Le marquis de Denonville, gouverneur de la Nouvelle-France, accuse son homologue de la Nouvelle-Angleterre, sir Edmund Andros, ou du moins ses subordonnés à Boston, d'avoir contribué à l'armement de Peterson. En fait, les populations du Rhode Island et de Salem assistèrent le pirate à l'insu des autorités locales. 
pour le Port Royal, sortirent du port de Chédabouctou, et allèrent le même jour mouiller dans le port de Campseau qui est à 7 lieues de là, à l'embouchure de la baie dite de Campseau, dans le fond de laquelle est Chédabouctou [...] .

La nuit du 9 au 10, l'équipage du navire, composé de 22 hommes, ne faisant point le quart parce qu'ils étaient extrêmement fatigués du travail des jours précédents [...]. Soixante hommes bien armés, qui étaient venus bien doucement dans un canot de guerre et des chaloupes à la faveur des îles pleines de bois qui sont dans ce port, sautèrent sur son bord et se saisirent aisément du capitaine et de l'équipage pendant que d'autres s'emparaient de la barque. Ils prirent aussi en même temps tous les pêcheurs qui étaient de ce côté-là, fatigués, endormis et sans arme, au nombre de 50, et même quelques Sauvages qui s'y trouvèrent, dont ils brisèrent les canots pour empêcher qu'ils ne donnassent avis à Chédabouctou de ce qui se passait.

Le 10 au matin, le commandant de Chédabouctou, le commis général et le missionnaire, venant dans une chaloupe pour expédier la barque pour le Port Royal et faire charger promptement le navire pour le renvoyer en France, tombèrent entre les mains de ces corsaires qui les arrêtèrent et les ramenèrent au navire de ces forbans qui était demeuré caché entre des îles hors le port, y entra.

[...] Le 11 après-midi, les corsaires mirent le commandant, le commis général, le missionnaire et soixante de leurs hommes dans le même canot et des chaloupes et regagnèrent droit à Chédabouctou où ils arrivèrent à minuit et, où étant entrés aisément, ils pillèrent pendant trois jours entiers et emportèrent tout ce qu'ils pouvaient dans des barques jusqu'au 15 au matin qu'ils s'en allèrent sans mettre le feu ni amener le commandant ni personne de l'habitation, où ils laissèrent pour 6 mois de vivres pour 70 personnes qui sont demeurés, qu'ils ne purent pas emporter et dont ils ne s'aperçurent pas.

[...] Dans le même temps que ces corsaires prirent le vaisseau Le St-Louis et la barque destinée pour le Port Royal par la Compagnie, ils prirent aussi, dans le même port de Campseau, une barque du nommé St-Aubin, habitant de l'Acadie, qui y était venu chercher du sel pour la pêche que la Compagnie l'avait excité de faire vers la rivière de Ste-Croix, entre celle de St-Jean et Pentagouët, pour laquelle on lui fournissait toutes choses. Le 22 août au matin, les corsaires sortirent du port de Campseau dans le navire Le St-Louis, emmenant encore avec eux 3 barques chargées et 3 chaloupes, les 50 pêcheurs de Campseau et les Sauvages. ${ }^{11}$

11 Raynald Laprise, " Descente d'un flibustier anglais en Acadie en 1688 », Les Cahiers de la Société historique acadienne, vol. 33, numéros 1 et 2 (mars-juin 2002), p. 33-40. 
Les écarts qui se dégagent de la comparaison entre le témoignage de Challe et les versions données par les autres documents soulèvent une interrogation de taille : quelle est l'origine ou la cause de ces divergences? S'agit-il d'erreurs occasionnées par des informateurs mal renseignés, ou s'agit-il plutôt d'un détournement volontaire inspiré par des mobiles quelconques? Comme nous nous appliquerons à le montrer dans les lignes qui suivent, différentes raisons nous portent à privilégier la piste d'un détournement délibéré des faits.

\section{Pourquoi ce glissement dans la fiction ${ }^{12}$ ?}

Se remémorant l'événement à deux moments différents, Challe poursuit à chaque fois un objectif précis. Dans le récit du Journal, il paraît évident que Challe veut mettre l'accent sur la poltronnerie de La Boulaye ${ }^{13}$. Dire que les canons n'étaient pas montés serait une façon de défendre le gouverneur et ses officiers en leur fournissant une excuse pour ne pas avoir opposé de résistance, ce qui n'est évidemment pas compatible avec le projet de Challe. Par contre, en disant que le "fort était bien garni de trente canons bien montés ", l'écrivain du roi met directement en cause la bravoure de La Boulaye, ce qui lui permet après coup de se présenter comme l'homme de la situation. Le portrait caricatural dont La Boulaye fait l'objet contraste tout à fait avec le récit du combat héroïque que Challe prétend avoir livré contre les Anglais - récit qui rappelle étrangement celui où l'une des figures idéalisées par Challe, en l'occurrence Duquesne, livre un combat héroïque contre des pirates Algériens. Dans l'entrée du 24 avril 1690, l'écrivain du roi rapporte le

12 La question qui se pose ici est de savoir pourquoi Challe a transposé dans le récit de la prise de 1688 un fait qui n'est survenu que deux ans plus tard. Faut-il y voir une " simple inadvertance de celui qui parle par ouï-dire »? Cela est fort possible. Mais les efforts déployés par Challe dans les deux comptes rendus pour démolir son prétendu rival indiquent qu'il s'agirait plutôt d'un désir délibéré d'accabler davantage La Boulaye en aggravant l'étendue du désastre.

13 La volonté de ruiner la réputation de La Boulaye se traduit par diverses occurrences. Notons d'abord cette tournure ironique utilisée par l'écrivain du roi : «Il fut pourtant, grâce à la vigilante conduite du gouverneur \& des officiers, pris " (Journal, p. 215). L'allusion faite à la prestation de La Boulaye pendant la campagne de Flandre vise aussi à souligner le ridicule de la façon dont il s'est fait prendre en Acadie : "Il avait du service, \& avait fort bien défendu le pont d'Avendin en Flandre contre le prince d'Orange en 1677, \& s'est fait prendre comme un sot » (Id, p. 215). 
discours où monsieur de Charmote vante les prouesses militaires du regretté capitaine. La ressemblance est troublante :

Qu'il avait été pris par les Algériens : que la vigoureuse résistance qu'il avait faite à quatre frégates, dont la moindre était aussi forte que celle qu'il montait, avait forcé ces barbares à respecter sa conduite, son intrépidité \& sa valeur, dans un combat si inégal; ne s'étant rendu qu'au quatrième abordage, blessé à quatre endroits, \& hors d'état de se défendre davantage, ayant perdu quarantedeux hommes de soixante-quinze dont son équipage était composé en sortant de La Rochelle. ${ }^{14}$

Dans la version des Mémoires, il semble que Challe poursuive un but différent. Son projet n'est pas de remettre en question la prouesse militaire de La Boulaye, mais de critiquer ses compétences en matière de logistique et de gestion. C'est pour cette raison qu'il dit que «les canons n'étaient même pas montés». En plus de cette affirmation par laquelle il veut souligner la négligence coupable du gouverneur, Challe énumère une série d'erreurs commises par ce dernier. Il lui reproche le peu de fortifications, l'absence de garde et une tendance exagérée aux plaisirs. Le récit des Mémoires est régi par la même logique contrastive qui régente le témoignage du Journal. Si dans le récit de la traversée vers les Indes, Challe oppose sa bravoure à la lâcheté de La Boulaye, dans les Mémoires, l'énumération des délits commis par ce dernier lui permet de se présenter comme fin connaisseur en matière de gestion des colonies.

La campagne de démolition menée contre le gouverneur ${ }^{15} s^{\prime}$ accompagne, en effet, d'une campagne visant à faire valoir les mérites de celui qui raconte. Parmi les éléments, vrais ou supposés, qui permettent cet étalage de soi se trouve la déclaration où Challe prétend que Seignelay lui a demandé de rédiger des mémoires sur la façon dont les choses se

14 Journal, p. 176.

15 La transformation délibérée des faits historiques pour motif de vengeance est un geste fréquent chez Challe. Entretenant des relations conflictuelles avec la famille de Chevery, il n'hésite pas à démolir le représentant de ce clan dans l'univers romanesque des Illustres Françaises, en présentant une peinture caricaturale sous les traits de Contamine, héros de la deuxième nouvelle. Sur ce point voir l'article de Marie-Laure Girou-Swiderski, "La véritable Angélique, un double de Robert Challe? ", RHLF, vol. 83, n"4, 1983, 531-569, ainsi que celui de Jacques Popin, "Le mystérieux P.P.A.P.D.L. ", Cahiers Lorrains, $3^{\mathrm{e}}$ trimestre 1982, 211-220. Les deux études identifient le Contamine des Illustres Françaises : il s'agit de Charles Duret de Chevery, président de chambre au parlement de Metz. 
passent en Acadie : «M. de Seignelay m’avait ordonné de prendre garde à tout, et de faire une espèce de journal secret pour le lui communiquer à mon retour ${ }^{16}{ }^{\prime}$. Cette affirmation qui fait du mémorialiste l'informateur privilégié du futur ministre de la marine est appuyée par une autre déclaration, plus osée, où Challe prétend que Seignelay, ayant apprécié ses remarques, en parla au roi qui voulut le voir. Par ces indications, Challe veut se peindre comme le successeur légitime de Bergier au poste de lieutenant du roi. C'est ce qui ressort, en tout cas, de cette affirmation où il se présente comme le candidat de Seignelay: «Bergier fut remercié de ses bons et agréables services, et $\mathrm{M}$. de Seignelay jeta les yeux sur moi pour lui succéder ${ }^{17}{ }^{1}$. Dans le récit que Challe présente de la prise de Chédabouctou se profile donc une rivalité, qui n'est peut-être que fictive, mais qui permet au mémorialiste de se peindre comme celui qui aurait pu sauver le comptoir et toute l'Acadie si on lui en avait donné l'occasion : « M. de Seignelay [...] m'avoua que si mes mémoires avaient été suivis cela ne serait point arrivé ${ }^{18} \gg$. Peu vraisemblable en elle-même, ${ }^{19}$ l'éventualité que Seignelay l'ait pressenti pour succéder à Bergier paraît assez fondée aux yeux de Challe pour qu'il se rêve, un moment, tel qu'il aurait voulu être: soldat inconnu qui aurait pu changer le cours de l'histoire, si les choses avaient tourné à son avantage.

L'épisode relatant les circonstances qui entourent la prise de Chédabouctou permet à Challe de minimiser sa part de responsabilité dans ce qu'il décrit comme étant le plus grand drame de sa vie, et de montrer du doigt les véritables auteurs de son malheur. Fort de sa plume et de l'imaginaire vers lequel le porte son ambition trop écoutée, Challe agit en tant que justicier qui tente de se faire réparation en se donnant une histoire à la hauteur de ses aspirations. Il veut prouver que, si l'occasion lui en avait été donnée, il aurait réussi là où d'autres, pourtant jugés supérieurs, ont failli. Mettre en récit des épisodes où sa destinée par-

16 Mémoires, p. 379.

17 Ibid., p. 380.

18 Ibid., p. 389.

19 Les témoignages officiels corroborent « les affirmations de Challe lui-même en montrant les importantes fonctions qu'il occupait à ce moment, malgré son jeune âge (il n'a alors que 24 ans) en raison de la situation délicate du huguenot Bergier, bien illustrée par la brutalité de la lettre de La Barre. On y voit aussi la grande estime où le tenaient les personnages officiels. Ceci légitime, jusqu'à un certain point, son espoir fallacieux de voir récompenser son zèle par l'octroi du poste de Bergier, une fois celui-ci remercié .» (Marie-Laure Girou-Swiderski, «Compte rendu » de la récente édition des Mémoires, Francophonies d'Amérique, nº, p. 5). 
ticulière coïncide avec l'histoire équivaut aux yeux de Challe à un jeu qui consiste à réparer les injustices du destin à son égard. Dès lors, quoi de plus légitime que d'opérer quelques embellissements occasionnels? A cet égard, son infidélité à l'histoire, et la propension qu'il a à la manipuler au gré de mobiles personnels, sont à considérer comme une revanche sur la vie, la rançon à payer pour que l'homme de mérite puisse coïncider avec l'image qu'il a de lui-même.

Les retouches que Challe apporte à son moi montrent que le témoignage sur soi est, chez lui, le fruit d'un drame qui découle de l'incompatibilité entre les mérites de l'individu et sa fortune. Le sentiment d'avoir mené une vie en deçà de ses espérances le conduit apparemment à favoriser les dimensions introspective et prospective du témoignage sur soi, plutôt que son vecteur rétrospectif. Plus qu'une simple reconstitution des jours anciens, le récit de vie tel que pratiqué par Challe est un moyen de faire appel contre les injustices du destin et de se donner, en prenant la postérité à témoin, une ligne de vie plus satisfaisante. La littérature du moi, ouverte aux intrusions de l'imaginaire, devient terre promise où le Je qui se dit peut enfin épouser l'image rêvée qu'il a de lui-même. Au pacte inaugural incitant quiconque veut faire œuvre autobiographique à rester fidèle à l'exactitude historique, Challe substitue un pacte d'alliance avec soi qui l'affranchit partiellement des contraintes littéraires entourant la mise en récit de certains épisodes de vie.

DRISS AISSAOUI

Université Dalhousie 